\title{
A HOMOTOPY ${ }^{+}$SOLUTION TO THE A-B SLICE PROBLEM
}

\author{
MICHAEL FREEDMAN AND VYACHESLAV KRUSHKAL \\ Dedicated to the memory of Tim Cochran
}

\begin{abstract}
The A-B slice problem, a reformulation of the 4-dimensional topological surgery conjecture for free groups, is shown to admit a link-homotopy ${ }^{+}$solution. The proof relies on geometric applications of the group-theoretic 2-Engel relation. Implications for the surgery conjecture are discussed.
\end{abstract}

\section{INTRODUCTION}

Four-dimensional surgery is known to work in the topological category for a class of good fundamental groups. This result was originally established in the simplyconnected case in [3], and it is currently known to hold for groups of subexponential growth and a somewhat larger class generated by these [4, 10, 15]. The A-B slice problem [5, 6] is a reformulation of the surgery conjecture for free groups, which is the most difficult case.

The A-B slice problem concerns decompositions of the 4-ball. The handle structure of a decomposition, interpreted as a Kirby diagram, gives rise to a stabilization of a given link $L$, see section 2 for a precise definition. In these terms, to show that $L$ is A-B slice one needs to find a stabilization and band-sums between the components so that the resulting link is slice. The Generalized Borromean Rings (GBRs) are a collection of links any coinitial subset of which is universal for surgery. In a recent work [7] we showed that GBRs have a coinitial subset admitting a link-homotopy solution to the A-B slice problem. In other words, given such a GBR there exists a stabilization and band sums so that the resulting link is homotopically trivial (htrivial) in the sense of Milnor [18]. Here we sharpen this result:

Theorem 1. The A-B slice problem for a coinitial collection of generalized Borromean rings, forming universal surgery problems, admits a (link-homotopy) ${ }^{+}$solution.

The Generalized Borromean Rings are the collection of links obtained from the Hopf link by (any non-trivial amount of) iterated ramified Bing doubling. There is a natural partial order on GBRs where more ramification and more Bing doubling means "less than", see figure 1.1. 

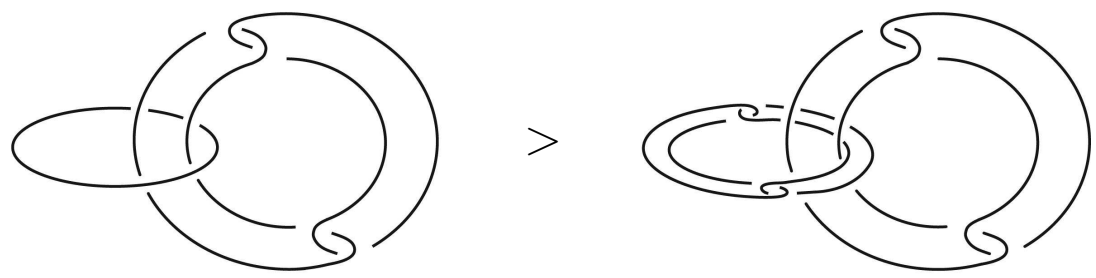

Figure 1.1. Partial order on Generalized Borromean Rings

An $n$-component link $K$ is called $h$-trivial ${ }^{+}$if each one of the $n$ links obtained by adding to $K$ a parallel copy of a single component is homotopically trivial. The extension from h-trivial to h-trivial ${ }^{+}$is of interest in part due to the theorem [11] that untwisted Whitehead doubles of h-trivial ${ }^{+}$links are topologically slice. This means that the strongest possible version of the A-B slice problem for such links has a solution. That is, given an $\mathrm{h}$-trivial ${ }^{+}$link, there exists a stabilization giving a relatively slice link. It is interesting to relate this to Theorem 1 which, starting with a GBR, finds a stabilization yielding an h-trivial ${ }^{+}$link. A natural question is whether these two stabilizations can be combined to give a genuine A-B slice solution for GBRs.

As discussed in [7], if a link homotopy solution can be sufficiently improved, this could lead to an affirmative resolution to the surgery conjecture for all groups. Our result may be seen as a step in this direction. In terms of Milnor's $\bar{\mu}$-invariants, 7 ] constructs a stabilization giving a link with trivial $\bar{\mu}$-invariants with non-repeating indices. Theorem 1 improves this to a link with trivial $\bar{\mu}$-invariants with at most two repeating indices. An interesting question is whether there exists a stabilization giving a link with all vanishing $\bar{\mu}$-invariants.

The proof of theorem 1 may be extended to give a link-homotopy ${ }^{+k}$ solution, where $+k$ means that any link obtained by adding a total of $k$ parallel copies of various components is homotopically trivial. However this gain comes at a price: the amount of Bing doubling in GBRs for which our methods give a link-homotopy ${ }^{+k}$ solution grows with $k$. (The simplest representative link has $2^{2 k}+1$ components.) It follows from grope height raising [9, Proposition 2.7] that such a collection of links, for a fixed $k$, is still universal for surgery, see [7, Proposition 4.1].

The novel ingredient in the construction of a link-homotopy solution in [7] is a geometric use of the group-theoretic 2-Engel relation $[[y, x], x]$, in conjunction with handle slides. An important algebraic feature underlying this construction is the fact that any 2-Engel group is nilpotent of a fixed class.

One natural way to approach the h-trivial ${ }^{+}$condition is to use the 3 -Engel relation. However, as discussed in [7], $n$-Engel relations for $n>2$ are generally not well understood. Instead, in this paper h-triviality $^{+}$is achieved through a systematic application of the 2-Engel relation. More precisely, we study links modulo a more 
subtle relation which may be termed " $(2,2)$-Engel". Roughly, a group element satisfies this relation if it is trivial modulo the 2-Engel relation in two different ways. h-triviality $^{+}$is seen to be a geometric consequence of this relation.

The background material on the A-B slice problem and the 2-Engel relation is summarized in sections 2, 3. Theorem 1 is proved in section 6 ,

\section{A-B SLICE LINKS AND THE RELATIVE SLICE PROBLEM}

This section gives a brief summary of the relevant background on the A-B slice problem, the reader is referred to [8], for a more detailed exposition. We also state the notion of a link-homotopy ${ }^{+}$solution to the A-B slice problem, used in theorem 1 .

A decomposition of $D^{4}$ is a pair of compact codimension zero smooth submanifolds with boundary $A, B \subset D^{4}$, satisfying conditions (1)-(3) below. Denote

$$
\partial^{+} A=\partial A \cap \partial D^{4}, \quad \partial^{+} B=\partial B \cap \partial D^{4}, \quad \partial A=\partial^{+} A \cup \partial^{-} A, \quad \partial B=\partial^{+} B \cup \partial^{-} B .
$$

(1) $A \cup B=D^{4}$

(2) $A \cap B=\partial^{-} A=\partial^{-} B$,

(3) $S^{3}=\partial^{+} A \cup \partial^{+} B$ is the standard genus 1 Heegaard decomposition of $S^{3}$.

The "attaching curves" $\alpha, \beta$ of $A, B$ (the cores of the solid tori $\partial^{+} A$, respectively $\partial^{+} B$ ) form the Hopf link in $S^{3}=\partial D^{4}$.

Given a $k$-component link $L=\left(l_{1}, \ldots, l_{k}\right) \subset S^{3}$, let $L^{\prime}=\left(l_{1}^{\prime}, \ldots, l_{k}^{\prime}\right)$ be its untwisted parallel copy.

Definition 2.1. A link $L$ is $A-B$ slice if there exist decompositions $\left(A_{i}, B_{i}\right)$, of $D^{4}$ and self-homeomorphisms $\phi_{i}, \psi_{i}$ of $D^{4}, i=1, \ldots, k$ such that all sets $\phi_{1} A_{1}, \ldots, \phi_{k} A_{k}$, $\psi_{1} B_{1}, \ldots, \psi_{k} B_{k}$ are disjoint and satisfy: $\phi_{i}\left(\partial^{+} A_{i}\right)$ is a tubular neighborhood of $l_{i}$ and $\psi_{i}\left(\partial^{+} B_{i}\right)$ is a tubular neighborhood of $l_{i}^{\prime}$, for each $i$.

The collection of $2 k$ manifolds $\left\{A_{i}, B_{i}\right\}$ are disjointly embedded into $D^{4}$ by the restrictions $\left.\phi_{i}\right|_{A_{i}},\left.\psi_{i}\right|_{B_{i}}$. Since these maps are restrictions of self-homeomorphisms of $D^{4}$, the embeddings are standard, in the sense that the complement $D^{4} \backslash \phi_{i}\left(A_{i}\right)$ is homeomorphic to $B_{i}$, and $D^{4} \backslash \psi_{i}\left(B_{i}\right)$ is homeomorphic to $A_{i}$. (This condition that the embeddings are standard is important: it was shown in [14] that any link with trivial linking numbers is weakly A-B slice, when this condition is omitted.) A version of this requirement, in the link-homotopy setting, is stated as condition 2.4,

The 4-dimensional topological surgery conjecture for free groups was reformulated in [5, 6] in terms of the A-B slice problem for the Generalized Borromean rings (GBRs), the collection of links formed from the Borromean rings by iterated ramified Bing doubling. An example is shown in figure 6.1. 
The notion of a link-homotopy ${ }^{+} \mathrm{A}-\mathrm{B}$ slice link relies on a choice of handle decompositions of the submanifolds $\left\{A_{i}, B_{i}\right\}$. We will analyze them in the context of the relative-slice problem, introduced in [8].

Given a decomposition $D^{4}=A \cup B$, without loss of generality it may be assumed [8] that each side $A, B$ has a handle decomposition (relative to the collar $S^{1} \times D^{2} \times I$ ) with only 1 - and 2-handles. Denote $A=\left(\partial^{+} A\right) \times I \cup H_{1} \cup H_{2}$. As usual, the 1 -handles will be considered as standard 2-handles $H_{1}^{*}$ removed from the collar, $A=\left(\partial^{+} A \times I \backslash H_{1}^{*}\right) \cup H_{2}$. The decompositions constructed in this paper (see section 6) have the property that the 2-handles $H_{2}$ of each side do not go through the handles $H_{1}^{*}$ of the same side. (See [7] for a discussion of this terminology.)

Suppose an $n$-component link $L$ is A-B slice, with decompositions $D^{4}=A_{i} \cup B_{i}, i=$ $1, \ldots, n$. Denote by $D_{0}^{4}$ a smaller 4 -ball obtained by removing from $D^{4}$ the collars on the attaching regions $\phi_{i}\left(\partial^{+} A_{i}\right), \psi_{i}\left(\partial^{+} B_{i}\right)$ of all submanifolds $\left\{\phi_{i}\left(A_{i}\right), \psi_{i}\left(B_{i}\right)\right\}$. Let $\mathcal{H}_{2}$ denote the 2 -handles of all these submanifolds, and $\mathcal{H}_{1}^{*}$ the 2 -handles removed from the collars, corresponding to the 1 -handles. Consider $\mathcal{H}_{1}^{*}$ as zero-framed 2handles attached to $D_{0}^{4}$. (See [7, Section 3.1] for more details.)

Consider the following two links $J, K$ in $S^{3}=\partial D_{0}^{4}$. $J$ denotes the attaching curves of the 2 -handles $\mathcal{H}_{2}$, and $K$ the attaching curves of the 2-handles $\mathcal{H}_{1}^{*}$. (Here $\mathcal{H}_{2}$ are 2 -handles contained in $D_{0}^{4}$, and $\mathcal{H}_{1}^{*}$ are attached to $D_{0}^{4}$ with zero framings along $K$.) We call the pair $(J, K)$ a stabilization of the original link $L$. The structure of the stabilization links, which is a consequence of the duality between the 1 - and 2-handles of the two sides of each decomposition, is shown in figure 2.1. In terms of the notation in the figure, $J=L \cup_{i} J_{i} \cup_{i} \widehat{K}_{i}, K=\cup_{i} K_{i} \cup_{i} \widehat{J}_{i}$.

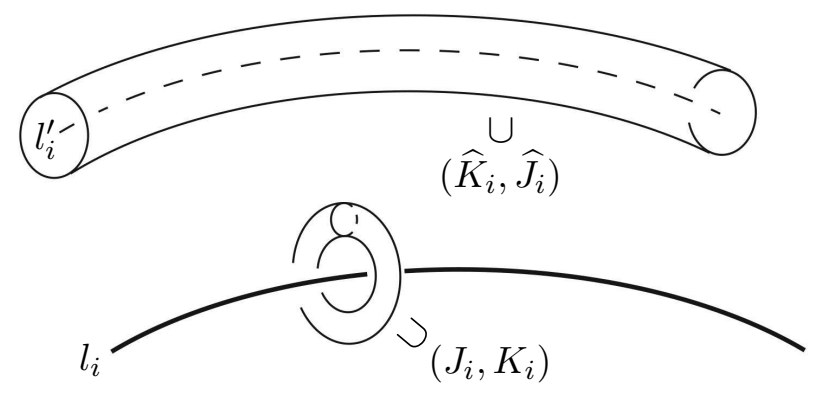

Figure 2.1. Stabilization corresponding to an A-B slice link $L=$ $\left\{l_{i}\right\}$ : link pairs $\left(J_{i}, K_{i}\right) \subset$ solid torus neighborhood of a meridian to $l_{i},\left(\widehat{K}_{i}, \widehat{J}_{i}\right) \subset$ solid torus neighborhood of a parallel copy $l_{i}^{\prime}$, and a diffeomorphism between the solid tori exchanging their meridian and longitude takes $K_{i}$ to $\widehat{K}_{i}$ and $J_{i}$ to $\widehat{J}_{i}$. 
Definition 2.2. A link pair $(J, K)$ in $S^{3}=\partial D_{0}^{4}$ is called relatively slice if the components of $J$ bound disjoint, smoothly embedded disks in the handlebody

$$
H_{K}=D_{0}^{4} \cup \text { zero-framed 2-handles attached along } K \text {. }
$$

If a link $L$ is A-B slice, the associated link pair $(J, K)$ is relatively slice. We now turn to the definition of a link-homotopy ${ }^{+}$solution to the A-B slice problem, referred to in the statement of theorem 1 .

Definition 2.3. A $k$-component link $L$ is link-homotopy ${ }^{+} A$-B slice if there exist decompositions $D^{4}=A_{i} \cup B_{i}, i=1, \ldots, k$ and handle decompositions of the submanifolds $A_{i}, B_{i}$ so that the associated relative-slice problem $(J, K)$ has a link-homotopy ${ }^{+}$ solution. In other words, in the context of definition 2.2 for each component $l$ of $J$ the link $J \cup l^{\prime}$ bounds disjoint maps of disks $\Delta$ in the handlebody $H_{K}$. Here $l^{\prime}$ denotes an untwisted parallel copy of $l$. Moreover, the disks $\Delta$ are subject to condition 2.4 below.

Recall that in the formulation of the A-B slice problem the disjoint embeddings of the manifolds $\left\{A_{i}, B_{i}\right\}$ are required to be standard, see the paragraph following definition 2.1. We formulate a version of this condition in the link-homotopy setting (see [7, Section 3.1] for a more detailed discussion):

Condition 2.4. Let $S$ be any submanifold in the collection $\left\{\phi_{i}\left(A_{i}\right), \psi_{i}\left(B_{i}\right)\right\}$. Then the maps of disks $\Delta$ for the components of $J \cup l^{\prime}$ corresponding to $S$ do not go through (are disjoint from the co-cores of) the 2-handles attached to $D_{0}^{4}$ along the components of $K$ corresponding to the same submanifold $S$.

\section{The Milnor group And the 2-Engel Relation}

We start by giving a brief overview of the Milnor group [18]. Let $\pi$ be a group normally generated by a fixed finite collection of elements $g_{1}, \ldots, g_{k}$. The Milnor group of $\pi$, defined with respect to a given normal generating set $\left\{g_{i}\right\}$, is defined by

$$
\left.M \pi=\pi /\left\langle\left\langle g_{i}, g_{i}^{y}\right] i=1, \ldots, k, y \in G\right\rangle\right\rangle .
$$

The Milnor group $M L$ of a link $L$ in $S^{3}$ is set to be the Milnor group $M \pi$ where $\pi=\pi_{1}\left(S^{3} \backslash L\right)$, defined with respect to meridians to the link components.

Denote by $F_{g_{1}, \ldots, g_{k}}$ the free group generated by the $\left\{g_{i}\right\}, i=1, \ldots, k$. The Magnus expansion

$$
M: F_{g_{1}, \ldots, g_{k}} \longrightarrow \mathbb{Z} \llbracket x_{1}, \ldots, x_{k} \rrbracket
$$

into the ring of formal power series in non-commuting variables $\left\{x_{i}\right\}$ is given by

$$
M\left(g_{i}\right)=1+x_{i}, M\left(g_{i}^{-1}\right)=1-x_{i}+x_{i}^{2}-x_{i}^{3} \pm \ldots
$$


The Magnus expansion induces an injective homomorphism

$$
M F_{g_{1}, \ldots, g_{k}} \longrightarrow R_{x_{1}, \ldots, x_{k}},
$$

into the quotient $R_{x_{1}, \ldots, x_{k}}$ of $\mathbb{Z} \llbracket x_{1}, \ldots, x_{k} \rrbracket$ by the ideal generated by all monomials $x_{i_{1}} \cdots x_{i_{k}}$ with some index occuring at least twice. Milnor's $\bar{\mu}$-invariants of a link are defined in terms of coefficients of the Magnus expansion [19].

Two links are link-homotopic if they are connected by a 1-parameter family of link maps where different components stay disjoint for all values of the parameter. If $L$, $L^{\prime}$ are link-homotopic then their Milnor groups $M L, M L^{\prime}$ are isomorphic. Moreover, a $k$-component link $L$ is homotopically trivial (h-trivial) if and only if $M L$ is isomorphic to the free Milnor group $M F_{m_{1}, \ldots, m_{k}}$. Equivalently, a link is h-trivial if and only if all its $\bar{\mu}$-invariants with non-repeating indices are trivial.

This paper concerns a stronger version of this equivalence relation. An $n$-component link $L$ is called $h$-trivial ${ }^{+}$if each one of the $n$ links obtained by adding to $L$ a parallel copy of a single component is homotopically trivial. A link is h-trivial ${ }^{+}$if and only if all its $\bar{\mu}$-invariants with at most two repeating indices are trivial.

3.1. 2-Engel groups. Given a group $\pi$, consider its lower central series defined by $\pi^{1}=\pi, \pi^{n}=\left[\pi^{n-1}, \pi\right]$. It is convenient to introduce a concise commutator notation

$$
\left[g_{1}, g_{2}, \ldots, g_{n}\right]:=\left[\left[\ldots\left[g_{1}, g_{2}\right], \ldots, g_{n-1}\right], g_{n}\right] \text {. }
$$

This paper concerns geometric applications of the 2-Engel relation $[[y, x], x]=1$, or equivalently $\left[x, x^{y}\right]=1$. A 2 -Engel group $\pi$ is a group satisfying this relation for all $x, y \in \pi$. Note the difference with the definition of the Milnor group (3.1) where this relation is imposed only on $x$ in a fixed set of normal generators.

The free Milnor group on $n$ generators $M F_{n}$ is nilpotent of class $n$ [18]. In contrast, the nilpotency class of 2-Engel groups is independent of the number of generators. This result, building on earlier work of Burnside [1], is due to Hopkins [12] (also see [16]):

Lemma 3.1. Any 2-Engel group is nilpotent of class $\leq 3$.

A proof in the context of the Milnor group is given in [7]. The following more precise statement will be useful for applications in the next section.

Corollary 3.2. [7, Corollary 2.3] Suppose $\pi$ is a group normally generated by $g_{1}, \ldots, g_{n}$. Let $g \in \pi^{k}$ be an element of the $k$-th term of the lower central series, $4 \leq k \leq n$. Then $g$ may be represented in the Milnor group $M \pi$ as a product of (conjugates of) $k$-fold commutators $C$ of the form $\left[h_{1}, \ldots, h_{k}\right]$ where two of the elements $h_{i}$ are equal to each other and to a product of two generators, $h_{j}=h_{m}=g_{i_{1}} g_{i_{2}}$ for some $j \neq m$, and each other element $h_{i}$ is one of the generators $g_{1}, \ldots, g_{n}$. 
We will refer to the commutators of the form $\left[h_{1}, \ldots, h_{k}\right]$ in the statement of Corollary 3.2 as almost basic commutators. (This term is meant to avoid confusion with commutators $\left[g_{i_{1}}, \ldots, g_{i_{k}}\right]$ which are usually called basic.)

Figure 3.1 shows examples of links which are a geometric realization (for $k=4$ ) of almost basic commutators. A central feature of these homotopically essential links is that a 0 -framed handle slide (in the notation of the figure, of the $z$-curve over the $y$-curve) gives a split link consisting of an unknot and a homotopically trivial link pictured in figure 6.2. These links will be used to construct decompositions of $D^{4}$ in section 6 .
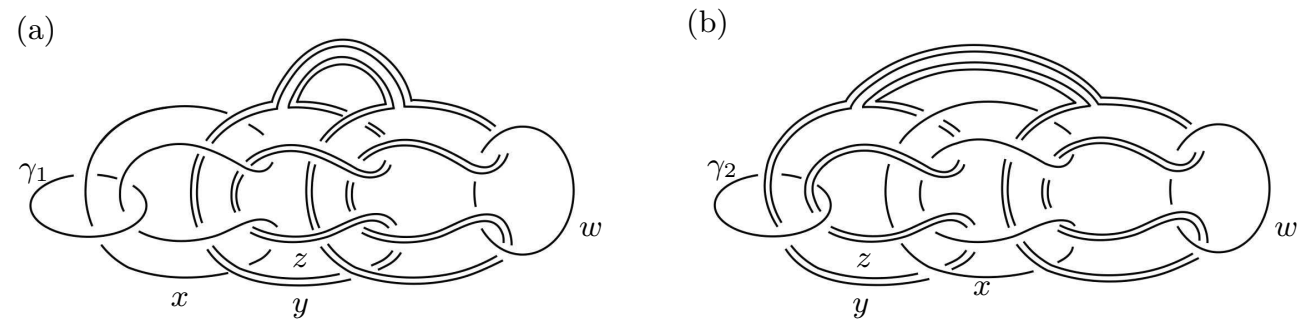

Figure 3.1. (a): $\gamma_{1}=[x, y z, y z, w], \quad(\mathrm{b}): \gamma_{2}=[y z, x, y z, w]$.

Section 6.2 will use the following variation of the statement of Corollary 3.2 .

Proposition 3.3. Let $\pi$ be a group normally generated by $g_{1}, \ldots, g_{n}$. Suppose $g \in \pi^{k}, 5 \leq k \leq n$. Then $g$ may be represented in the Milnor group $M \pi$ as a product of (conjugates of) $k$-fold almost basic commutators $\left[h_{1}, \ldots, h_{k}\right]$, where the first element $h_{1}$ is a generator $g_{i}$ (and not a product of two generators).

Proof. To be specific, consider an element $g \in \pi^{k}$, where $k=5$. (The proof for any $k \geq 5$ is analogous.) Considered as an element of $(M G)^{5}, g$ equals a product of conjugates of 5 -fold basic commutators $\left[g_{i_{1}}, \ldots, g_{i_{5}}\right]$ with distinct indices. According to Corollary $3.2,\left[g_{i_{2}}, \ldots, g_{i_{5}}\right]$ is a product of conjugates of almost basic commutators $C$. Using commutator identities (5.3), $\left[g_{i_{1}}, \ldots, g_{i_{5}}\right]$ is then seen to be a product of conjugates of elements of the form $\left[g_{i_{1}}, C\right]$.

Links corresponding to 5 -fold commutators as in the statement of proposition 3.3 are shown in figure6.4. (Note that the component labeled $g_{i}$ in the figure is not involved in band sums forming the links.)

\section{A motivating example}

Before giving a formal proof of theorem 1 we illustrate the idea underlying h-triviality ${ }^{+}$ in the set-up in figure 4.1. Start with the Borromean rings and let $T_{1}, T_{2}$ denote solid torus neighborhoods of two of the components. Let $L_{i} \subset T_{i}$ be two links embedded in these solid tori. Denote by $\Lambda_{i}$ a meridian of $T_{i}$ : a curve in $\partial T_{i}$ bounding a disk in $T_{i}$, figure 4.1 , 


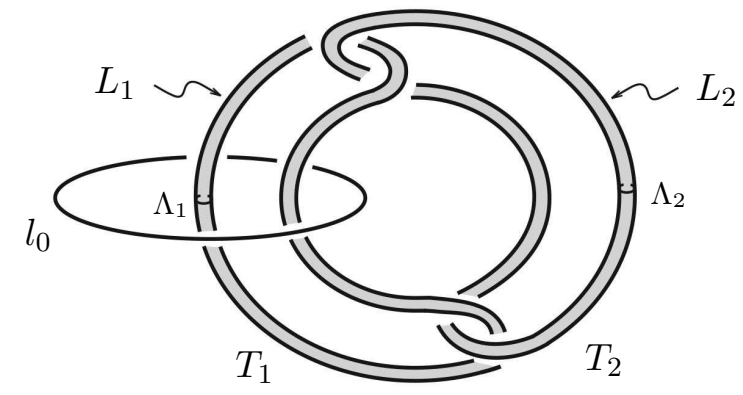

Figure 4.1. The link $L=l_{0} \cup L_{1} \cup L_{2}$ in lemma 4.1.

Lemma 4.1. Consider the link $L=l_{0} \cup L_{1} \cup L_{2}$, figure 4.1, Suppose that for each $i=1,2$,

(1) $L_{i} \cup \Lambda_{i}$ is h-trivial, and

(2) $L_{i}$ is h-trivial ${ }^{+}$in $S^{3}$, where $L_{i} \subset T_{i} \subset S^{3}$ and $T_{i} \subset S^{3}$ is the standard inclusion.

Then $L$ is h-trivial ${ }^{+}$.

Figure 4.2 shows an example of a link $L_{i}$ in the solid torus satisfying the assumption in lemma 4.1; the Whitehead double of the core of the solid torus, and a parallel copy. (Note that in this example $L_{i} \cup \Lambda_{i}$ is h-trivial, but $L_{i}$ is not h-trivial in the solid torus.) Other examples are given by the links in figure 6.2, where the three components on the right form the link in the solid torus = complement in $S^{3}$ of the left-most component.

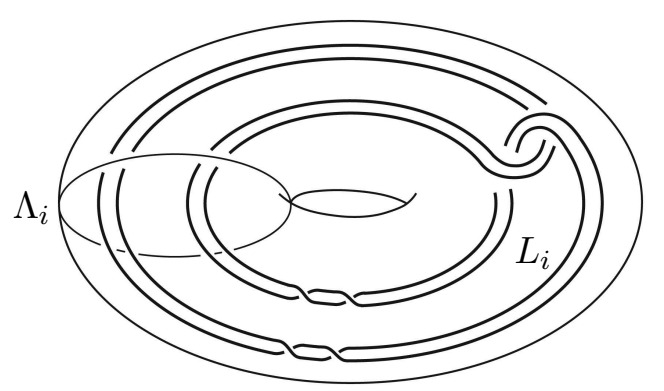

Figure 4.2. An example of a link $L_{i}$ in the solid torus satisfying the assumptions in lemma 4.1 .

Proof of lemma 4.1. There are two separate cases to consider: when a parallel copy of a component of $L_{1} \cup L_{2}$ is added, and when a parallel copy of $l_{0}$ is added. First consider $L^{\prime}:=L \cup$ parallel copy $l_{1}^{\prime}$ of a component of $L_{1}$. We start with a geometric argument to show that $L^{\prime}$ is h-trivial. The steps below are labeled for referencing in follow-up sections. 
(1) The link $L$ may be built starting with $L_{2} \cup \Lambda_{2}$ as follows: Bing double $\Lambda_{2}$, denote one of the resulting components by $l_{0}$ and insert $L_{1}$ in a tubular neighborhood of the other component. Pictured this way, $l_{0} \cup L_{1}$ is contained in a tubular neighborhood of $\Lambda_{2}$. Consider a link null-homotopy of $L_{2} \cup \Lambda_{2}$ and extend it to $L_{2} \cup\left(l_{0} \cup L_{1}\right)$. Selfintersections of $\Lambda_{2}$ during the link-homotopy are implemented by self-intersections of $l_{0}$. This gives a link-homotopy of $L$ where the components of $L_{1}$ have no selfintersections, so the same argument goes through when a parallel copy $l_{1}^{\prime}$ is added to $L_{1}$. The assumption (2) of the lemma completes the proof that $L^{\prime}$ is h-trivial. h-triviality of $L \cup$ parallel copy of a component of $L_{2}$ is established analogously.

(2) Now we give another, algebraic, proof that $L^{\prime}$ is h-trivial. This argument will be applicable in the more general setting of theorem 11. Abusing the notation, let $l_{0}, \Lambda_{i}$ refer to based loops. Then

$$
l_{0}=\left[\Lambda_{1}, \Lambda_{2}\right]
$$

where

$$
\Lambda_{1}=\Lambda_{2}=1 \in M \pi_{1}\left(S^{3} \backslash\left(L_{1} \cup L_{2}\right)\right)
$$

The statement (4.2) follows from the fact that $\left(L_{1} \cup \Lambda_{1}\right)$ and $\left(L_{2} \cup \Lambda_{2}\right)$ form a split link in $S^{3} \times 1$ which is homotopically trivial by the assumption (1) of the lemma.

Therefore every monomial (other than 1 ) in the Magnus expansion of $l_{0}$ has two sets of repeated variables: one pair corresponding to a component of $L_{1}$ and another pair corresponding to a component of $L_{2}$. This implies that the link remains h-trivial when a parallel copy is added to one component of either $L_{1}$ or $L_{2}$.

(3) Now consider $L \cup l_{0}^{\prime}$, where $l_{0}^{\prime}$ is a parallel copy of $l_{0}$. Note that there exist maps of disks $\Delta$ into $D^{4}$ bounded by $L_{1} \cup L_{2}$ and a capped punctured torus $T^{c}$ bounded by $l_{0}$ in $D^{4}$ such that all disks and $T^{c}$ are pairwise disjoint. The body of $T^{c}$ is an embedded genus 1 surface bounded by $l_{0}$ in $S^{3} \backslash\left(T_{1} \cup T_{2}\right)$, with a symplectic basis of curves isotopic to $\Lambda_{1}, \Lambda_{2}$. Extend $L_{1} \cup \Lambda_{1} \cup L_{2} \cup \Lambda_{2}$ by a product in a collar $S^{3} \times I \subset D^{4}$, where $S^{3} \times 0$ is identified with $\partial D^{4}$. Then $L_{1} \cup \Lambda_{1}$ and $L_{2} \cup \Lambda_{2}$ form a split link in $S^{3} \times 1$ which is h-trivial by the assumption (1) of the lemma. The null-homotopies for $\Lambda_{1}, \Lambda_{2}$ give the caps for $T^{c}$. Contraction/push-off [9, Section 2.3] applied to $T^{c}$ and its parallel copy give disjoint maps of disks for $l_{0}, l_{0}^{\prime}$ in the complement of the disks $\Delta$ bounded by $L_{1} \cup L_{2}$. This concludes the construction of disjoint disks for all components of $L \cup l_{0}^{\prime}$.

Remark. Contraction/push-off in part (3) of the proof, if desired, could be iterated to show that $L \cup$ (any given number of parallel copies of $l_{0}$ ) is a homotopically trivial link. 


\section{A lemma in COMmutator CAlculus.}

Lemma 4.1 above illustrates the idea that the improvement from a homotopy solution in [7] to a homotopy ${ }^{+}$solution will follow from allowing two "parallel channels" or "two participants in a commutator" by which an element can die. More precisely, the key features of the link $l_{0} \cup L_{1} \cup L_{2}$ in figure 4.1 are the expression (4.1) for $l_{0}$, subject to (4.2), and the fact that $L_{1} \cup L_{2}$ is h-trivial ${ }^{+}$. The link that will come up in the proof of theorem 1 is more general than the basic example in lemma 4.1 , This section develops the relevant algebraic framework which generalizes Corollary 3.2 using the main features described above.

Note that the representation of $g$ in Corollary 3.2 as a product of conjugates of almost basic commutators holds in the Milnor group, in general it is not valid in the group $\pi$. For the purpose of proving Theorem 1 it is insufficient to work modulo the Milnor relation, a more subtle equivalence relation is needed.

Let $\pi$ be a group normally generated by a fixed set of elements $\left\{g_{1}, \ldots, g_{n}\right\}$. Motivated by the defining relations (3.1) of the Milnor group and by the equations (4.1), (4.2), consider commutators of the form

$$
\left[\left[g_{i}, g_{i}^{y_{1}}\right]^{z_{1}},\left[g_{j}, g_{j}^{y_{2}}\right]^{z_{2}}\right]
$$

where $1 \leq i, j \leq n$, and $y_{k}, z_{k}$ are arbitrary elements of $\pi$. The notation $f \equiv g$ for two elements $f, g \in \pi$ will indicate that $f \cdot g^{-1}$ is in the normal subgroup generated by the elements (5.1). Technically we will not consider quotients of groups by these relations, rather (5.1) will be used in section 6 to construct specific h-trivial ${ }^{+}$links.

The following lemma establishes a version of Corollary 3.2 in the setting of the relations (5.1). A useful fact about the lower central series to keep in mind is that $\left[\pi^{p}, \pi^{q}\right] \subset \pi^{p+q}$.

Lemma 5.1. Let $\pi$ be a group normally generated by $\left\{g_{1}, \ldots, g_{n}\right\}$. Fix $k \geq 4$ and consider a commutator $[\alpha, \beta]$ where $\alpha, \beta$ are both elements of the $k$ th term of the lower central series $\pi^{k}$. Then there exists $W \in \pi^{2 k}$ such that

(1) $[\alpha, \beta] \equiv W$, and

(2) $W$ equals in $\pi$ a product of conjugates of elements of the form $[C, \beta],\left[\alpha, C^{\prime}\right]$, $\left[C, C^{\prime}\right]$ where $C, C^{\prime}$ are almost basic commutators $\left[h_{1}, \ldots, h_{k}\right]$ of the form introduced in Corollary 3.2. Moreover, for $k \geq 5$ the almost basic commutators $C, C^{\prime}$ may be assumed to be of the form introduced in Proposition 3.3 .

Proof of lemma 5.1. Let $\alpha^{\prime}, \beta^{\prime} \in \pi$ denote products of conjugates of almost basic commutators representing $\alpha, \beta$, given by Corollary 3.2 ,

$$
\alpha \cdot\left(\alpha^{\prime}\right)^{-1}=1 \in M \pi, \beta \cdot\left(\beta^{\prime}\right)^{-1}=1 \in M \pi .
$$


Note that in general this does not imply $[\alpha, \beta] \equiv\left[\alpha^{\prime}, \beta^{\prime}\right]$. Recall the basic commutator identities, cf. [17, Theorem 5.1]:

$$
[x, y z]=[x, z] \cdot[x, y]^{z}, \quad[x z, y]=[x, y]^{z} \cdot[z, y] .
$$

It follows from (5.2) that $\alpha \cdot\left(\alpha^{\prime}\right)^{-1}, \beta \cdot\left(\beta^{\prime}\right)^{-1}$ equal products of conjugates of the defining Milnor relations (3.1):

$$
\alpha \cdot\left(\alpha^{\prime}\right)^{-1}=\prod_{i}\left[g_{i}, g_{i}^{x_{i}}\right]^{y_{i}}, \beta \cdot\left(\beta^{\prime}\right)^{-1}=\prod_{j}\left[g_{j}, g_{j}^{z_{j}}\right]^{w_{j}}
$$

where $x_{i}, y_{i}, z_{j}, w_{j}$ are arbitrary elements of $\pi$. Substituting these expressions for $\alpha \cdot\left(\alpha^{\prime}\right)^{-1},\left(\beta^{\prime}\right)^{-1} \cdot \beta$ and using (5.3) , the commutator $\left[\alpha \cdot\left(\alpha^{\prime}\right)^{-1},\left(\beta^{\prime}\right)^{-1} \cdot \beta\right]$ is seen to equal a product of conjugates of elements of the form (5.1). In other words,

$$
\left[\alpha \cdot\left(\alpha^{\prime}\right)^{-1},\left(\beta^{\prime}\right)^{-1} \cdot \beta\right] \equiv 1 .
$$

Again using (5.3),

$$
\left[\alpha \cdot\left(\alpha^{\prime}\right)^{-1},\left(\beta^{\prime}\right)^{-1} \cdot \beta\right]=[\alpha, \beta]^{\gamma_{1}} \cdot\left[\left(\alpha^{\prime}\right)^{-1}, \beta\right] \cdot\left[\alpha,\left(\beta^{\prime}\right)^{-1}\right]^{\gamma_{2}} \cdot\left[\left(\alpha^{\prime}\right)^{-1},\left(\beta^{\prime}\right)^{-1}\right]^{\gamma_{3}}
$$

for some $\gamma_{i} \in \pi$, determined by (15.3). Set $W \in \pi$ to be (a conjugate of) the inverse of the product of the three right factors in (5.5):

$$
\left.W:=\left(\left(\left[\left(\alpha^{\prime}\right)^{-1}, \beta\right] \cdot\left[\alpha,\left(\beta^{\prime}\right)^{-1}\right]^{\gamma_{2}} \cdot\left[\left(\alpha^{\prime}\right)^{-1},\left(\beta^{\prime}\right)^{-1}\right]^{\gamma_{3}}\right]\right)^{-1}\right)^{\gamma_{1}{ }^{-1}} .
$$

It follows from (5.4), (5.5) that

$$
[\alpha, \beta] \equiv W
$$

An application of the basic commutator identities [17, Theorem 5.1],

$$
\left[x, y^{-1}\right]=[y, x]^{y^{-1}},\left[x^{-1}, y\right]=[y, x]^{x^{-1}},
$$

allows one to eliminate the negative exponents of $\alpha^{\prime}, \beta^{\prime}$ in the definition (5.6) of $W$, thereby expressing $W$ in the form required in the statement (2) of the lemma.

The proof of theorem 1 in section 6 will require the following extension of lemma 5.1 .

Lemma 5.2. Let $\pi$ be a group normally generated by $\left\{g_{1}, \ldots, g_{n}\right\}$. Fix $k \geq 4$, and suppose $g \in \pi$ is of the form

$$
g=\left[\left[\alpha_{1}, \beta_{1}\right],\left[\alpha_{2}, \beta_{2}\right]\right],
$$

where each element $\alpha_{i}, \beta_{i}$ is in $\pi^{k}$. Then there exist $W_{1}, W_{2} \in \pi^{2 k}$, satisfying the condition (2) in Lemma 5.1, such that $g \equiv\left[W_{1}, W_{2}\right]$. 
Proof. An application of lemma 5.1 to $\left[\alpha_{i}, \beta_{i}\right]$ gives $W_{1}, W_{2} \in \pi^{2 k}$ such that $\left[\alpha_{i}, \beta_{i}\right] \equiv$ $W_{i}, i=1,2$.

Observe that given $x, y, z \in \pi, x \equiv y$ implies $[x, z] \equiv[y, z]$. Indeed, $x \equiv y$ means that $x \cdot y^{-1}$ is in the normal subgroup generated by the elements (5.1). Denote this normal subgroup by $N$. Then $x^{z} \cdot\left(y^{z}\right)^{-1}=\left(x y^{-1}\right)^{z} \in N$, so $x^{z} \equiv y^{z}$. Therefore $[x, z] \cdot[y, z]^{-1}=x \cdot\left(x^{-1}\right)^{z} \cdot\left(y \cdot\left(y^{-1}\right)^{z}\right)^{-1}=x \cdot\left(x^{-1}\right)^{z} \cdot y^{z} \cdot y^{-1}$ is also in $N$.

It follows that

$$
g=\left[\left[\alpha_{1}, \beta_{1}\right],\left[\alpha_{2}, \beta_{2}\right]\right] \equiv\left[W_{1},\left[\alpha_{2}, \beta_{2}\right]\right] \equiv\left[W_{1}, W_{2}\right] .
$$

\section{Proof of theorem 1}

As discussed in the introduction (also see [7, Proposition 4.1]) any coinitial subset of the Generalized Borromean Rings forms a collection of links universal for surgery. A homotopy A-B slice solution in [7] applies to links obtained from the Hopf link by keeping one of its components $l_{0}$ intact and Bing doubling the other components at least twice, see figure 6.1 for an example of such a link. A homotopy ${ }^{+}$solution constructed in sections 6.1- 6.2 applies to a collection of higher Bing-doubled links (still universal for surgery).

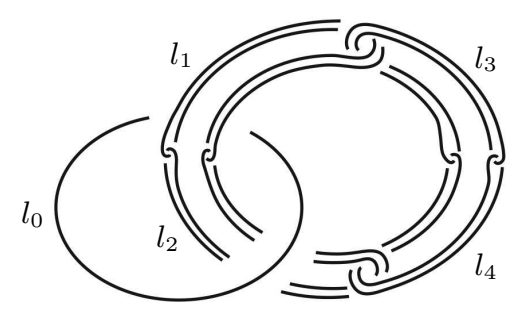

FiguRE 6.1. A link in the collection of GBRs.

The decompositions $D^{4}=A_{i} \cup B_{i}$ for all link components other than the fixed component $l_{0}$ are set to be the trivial decomposition, $A_{i}=2$-handle and $B_{i}=$ collar on the attaching curve $\beta_{i}$. Similarly to [7, the entire complexity of the construction is in the decomposition $D^{4}=A_{0} \cup B_{0}$ for the component $l_{0}$. $A_{0}$ will be defined to be the collar $l_{0} \times D^{2} \times I$ with a single 2-handle attached to the core of the solid torus $l_{0} \times D^{2} \times\{1\}$, and many 1-handles governed by the algebraic outcome of lemma 5.1, as explained below. In terms of figure 2.1 (where the index $i$ is understood to equal 0 ) the 2 -handle is attached to the curve labeled $l_{i}, J_{i}$ is empty since there are no other 2-handles, and $K_{i}$ is the Kirby diagram representation of the 1-handles. Correspondingly, the other side $B_{0}$ of the decomposition has no 1-handles ( $\widehat{J}_{i}$ in the figure is empty) and the attaching curves of its 2-handles form the link $\widehat{K}_{i}$. 
The link-homotopy solution in [7] uses a geometric implementation of Corollary [3.2, where each almost basic commutator of the form $\left[h_{1}, \ldots, h_{k}\right]$ is realized by a standard link illustrated in figure 3.1. More precisely, building blocks in the construction of the link $K_{0}$, describing the 1 -handles of $A_{0}$, are shown in figure 6.2, These are $h$-trivial counterparts of the links in figure 3.1 where one of the two parallel curves labeled $y, z$ is removed.
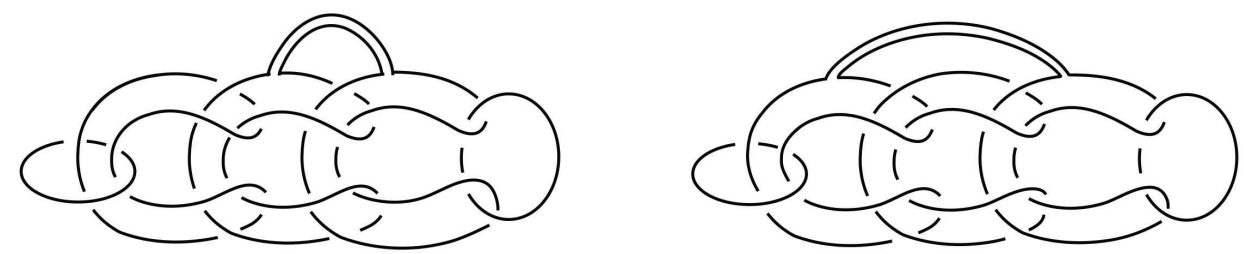

FiguRE 6.2. Links in figure 3.1, with one of the parallel components $y, z$ removed.

A key point, using the terminology of definition 2.2, is that $K_{0}$ is the attaching link for 0 -framed 2-handles attached to $D_{0}^{4}$, and parallel copies of each component bound disjoint copies of the core of the attached 2-handle. The links in figure 3.1 then may be recovered from links in figure 6.2 by adding the relevant parallel copy.

The homotopy solution from [7, Section 4] is schematically shown in Figure 6.3 (a). A modification of this construction, needed for the proof of theorem 1, is introduced in section 6.1. The theorem is then proved in three steps. The proof of h-triviality ${ }^{+}$ with respect to the components of $L$, band-summed with parallel copies of $K_{0}$, relies on lemma 5.1. h-triviality ${ }^{+}$with respect to the components of $\widehat{K}_{0}$ uses lemma 5.2, Finally, h-triviality ${ }^{+}$with respect to $l_{0}$ is analyzed in section 6.2 .

6.1. Construction of the stabilization. Let $\alpha_{1}, \beta_{1}, \alpha_{2}, \beta_{2}$ denote meridians to the components $l_{1}, \ldots, l_{4}$ of the link in figure 6.1. Consider the link $\left(l_{0}, L\right)$ in figure 6.3 (b), obtained by Bing doubling twice the components $l_{1}, \ldots, l_{4}$. Then $\alpha_{i}, \beta_{i}$ represent 4 -fold commutators in the meridians of $L$. The commutators $\left[\alpha_{1}, \beta_{1}\right],\left[\alpha_{2}, \beta_{2}\right]$ are meridians of the solid tori as indicated in figure 6.3 (b). The argument applies to GBRs that are more Bing-doubled and ramified; to be concrete we focus here on this simplest representative link. This section constructs:

- a stabilization of $l_{0}$ : a link $K_{0}$ in a solid torus linking $l_{0}$, and

- a band-sum $L^{\sharp}$ of $L$ with $\left(K_{0} \cup\right.$ parallel copies).

The following properties will be established:

- The link $K_{0}$ is h-trivial ${ }^{+}$in the solid torus,

- $\left(l_{0} \cup L^{\sharp}\right)$ is h-trivial ${ }^{+}$with respect to adding a parallel copy of a component of $L^{\sharp}$. 

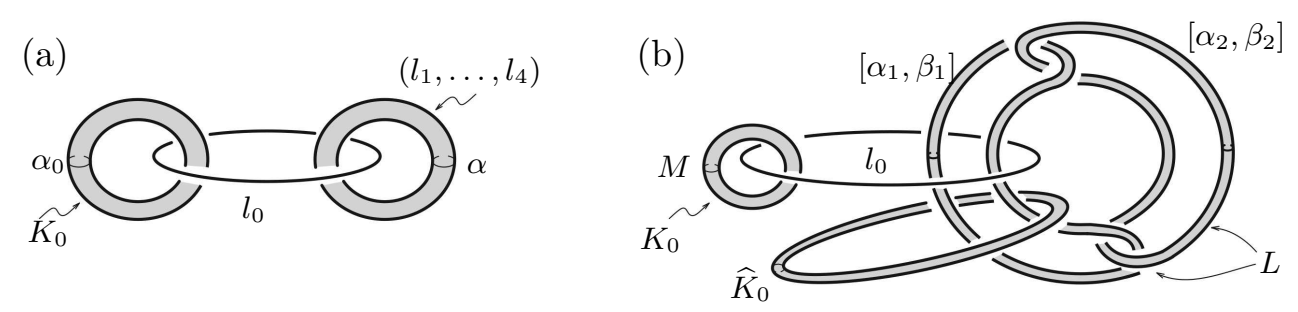

FiguRE 6.3.

(a) A schematic representation of the link-homotopy A-B slicing in [7], where $\left(l_{0}, \ldots, l_{4}\right)$ is the GBR is figure 6.1. (The link $\widehat{K}_{0}$, and also bands connecting $l_{2}, \ldots, l_{4}$ with $K_{0}$ are not shown.)

(b) The modified stabilization $K_{0}$, constructed in section 6.1, for the GBR $\left(l_{0}, L\right)$. Here $L$ is obtained by Bing doubling twice the components $l_{1}, \ldots, l_{4}$ of the link in figure 6.1.

The remaining step, homotopy triviality ${ }^{+}$with respect to a parallel copy of $l_{0}$ will be established in section 6.2.

To begin the construction, consider $\pi:=\pi_{1}\left(S^{3} \backslash L\right)$. L is the unlink, $\pi$ is the free group, and $l_{0}$ is an element of the form $\left[\left[\alpha_{1}, \beta_{1}\right],\left[\alpha_{2}, \beta_{2}\right]\right]$ as in equation (5.10). In the concrete example discussed above, each $\alpha_{i}, \beta_{i}$ is a 4 -fold commutator. (More generally, the argument applies to higher Bing doubles where $\alpha_{i}, \beta_{i}$ are $k$-fold commutators, $k \geq 4$. To obtain the argument in full generality, one replaces " 4 " (respectively "3") with " $k$ " (respectively " $k-1$ ") in each reference to the length of commutators or the number of link components in this section.) According to the statement (2) of lemma 5.1, each element $W_{1}, W_{2}$ in (5.10) is a product of conjugates of the elements of the form

$$
\left[C, \beta_{i}\right],\left[\alpha_{i}, C^{\prime}\right],\left[C, C^{\prime}\right],
$$

where $C, C^{\prime}$ are almost basic commutators as in Corollary 3.2. The stabilizing link $K_{0}$ will be constructed as a geometric analogue of the commutator $\left[W_{1}, W_{2}\right]$ in (5.10). Specifically, consider the Bing double of a meridian to $l_{0}$, and thicken the two resulting components to solid tori $T_{1}, T_{2}$. The link in the solid torus $T_{i}$ will be constructed to correspond to the commutator $W_{i}, i=1,2$. In the concrete example under discussion, the link $L$ is symmetric and the links constructed in $T_{1}, T_{2}$ are going to be identical. (In general, these links depend on the elements $W_{1}, W_{2}$ in section 5.2.)

Consider several copies of the core of each solid torus $T_{1}, T_{2}$, one for each factor in the statement (2) of lemma 5.1. For each element of the form (6.1) in the expression for $W_{i}$, take a Bing double of the corresponding copy of the core of $T_{i}$ and thicken the two resulting components to solid tori. Next we define links $K^{\prime}, K^{\prime \prime}$, geometrically representing the given elements (6.1) and insert them into these solid tori. For each element of the form $\left[C, \beta_{i}\right]$ consider a link of the type shown in figure [6.2, 
corresponding to the almost basic commutator $C$. More precisely, $K^{\prime}$ consists of three components on the right in a link in figure 6.2, considered in the solid torus complement of the leftmost component. Let $K^{\prime \prime}$ be the iterated Bing double of the core of the solid torus corresponding to the 4 -fold commutator $\beta_{i}$. The analogous pair of links $\left(K^{\prime}, K^{\prime \prime}\right)$ is created for each factor of the form (6.1) in the expression for $W_{i}$, completing the construction of $K_{0}$.

The construction in the preceding paragraph is a generalization of that in [7, Section 4], in particular see figure 4.3 in that reference. The stabilization in [7] was defined in terms of almost basic commutators $C$, while here we have links corresponding to commutators (6.1).

Now we construct the band sum $L^{\sharp}$, as promised at the beginning of section 6.1, For each constituent link of $K_{0}$ of the type in figure 6.2 add a parallel copy to one of the components to recreate a link as in figure 3.1. In the relative slice setting these parallel copies bound disjoint disks in the zero-framed 2-handles attached to $D_{0}^{4}$ along $K_{0}$. To find a homotopy ${ }^{+}$solution to the relative-slice problem, $L$ will be band-summed with the components of $K_{0}$ and their parallel copies. In the homotopy solution in [7] the choice of bands was immaterial. This was due to the fact that all commutators in the construction were of maximal length, so conjugation did not affect calculations in the Milnor group. The only relevant constraint for a homotopy solution was $l_{0}=\alpha \cdot\left(\alpha_{0}\right)^{-1}=1 \in M \pi_{1}\left(S^{3} \backslash L\right)$, in the notation of figure 6.3 (a). (The equality $l_{0}=\alpha \cdot\left(\alpha_{0}\right)^{-1}$ was established using additivity of $\bar{\mu}$-invariants [2, 13], or by directly reading off the element represented by $l_{0}$ in the Milnor group [7, Proof of theorem 1].) The homotopy ${ }^{+}$problem is more sensitive to the choice of bands.

Suppose the bands in the definition of $L^{\sharp}$ could be chosen so that the meridian $M$ of the solid torus in figure 6.3 (b) precisely matched the element $\left[W_{1}, W_{2}\right]$ in (5.10). Then in $\pi$ one would have $l_{0}=\left[\left[\alpha_{1}, \beta_{1}\right],\left[\alpha_{2}, \beta_{2}\right]\right] \cdot\left[W_{1}, W_{2}\right]^{-1} \equiv 1$, see (5.10). In this case the h-triviality ${ }^{+}$with respect to parallel copies of $L^{\sharp}$ is proved exactly the same way as in part (2) of the proof of lemma 4.1.

In fact, to establish h-triviality ${ }^{+}$with respect to parallel copies of $L^{\sharp}$ it suffices to choose bands so that $M$ suitably approximates $W$. Recall that each $W_{1}, W_{2}$ is a product of conjugates of the elements (6.1). The Milnor group $M \pi=M \pi_{1}\left(S^{3} \backslash L\right)$ is nilpotent of class equal to the number of components of $L$. For any choice of bands, each commutator (6.1) in the expression for $W_{i}$ is of maximal length in the power series of non-repeating monomials. Considering the Magnus expansion (3.2) of the free group $\pi$, note that only the homology class of conjugating elements is relevant. Indeed, suppose elements $W_{1}^{\prime}, W_{2}^{\prime}$ are created by some conjugating elements that agree homologically with those defining $W_{i}$ in Lemma 5.1, Then each term (other than 1) in the Magnus expansion of $\left[W_{1}, W_{2}\right] \cdot\left[W_{1}^{\prime}, W_{2}^{\prime}\right]^{-1}$ contains either three copies of a variable, or two pairs of different repeated variables. In either case adding a parallel copy preserves the condition of being h-trivial. Finally, choose arcs connecting 
$L$ with the relevant components of $K_{0}$ and its parallel copies, homologically matching the conjugating elements. Perform the band sums along these arcs; such operations do not interfere with each other since only homological information is relevant. This establishes h-triviality ${ }^{+}$with respect to components of $L^{\sharp}$.

It remains to show that the link $K_{0}$ is h-trivial ${ }^{+}$in the solid torus (or equivalently that $\widehat{K}_{0}$ is h-trivial ${ }^{+}$in the solid torus). Note that each link in the construction above, corresponding to an almost basic commutator $C$, is h-trivial in the solid torus. These links consist of three components on the right in a link in figure 6.2. considered in the solid torus complement of the leftmost component. The construction of $K_{0}$ was based on the commutator $\left[W_{1}, W_{2}\right]$ in (5.10), and both $W_{1}, W_{2}$ are products of conjugates of the elements of the form (6.1). $K_{0}$ was defined as the union of links in the two solid tori $T_{1}, T_{2}$ (whose cores are the Bing double of a meridian to $l_{0}$ ). The link in $T_{i}$ for each $i=1,2$, is h-trivial in the solid torus, therefore $K_{0}$ is h-trivial ${ }^{+}$ in the solid torus.

6.2. h-triviality ${ }^{+}$with respect to $\mathbf{l}_{0}$. Finally, consider the link $l_{0} \cup l_{0}^{\prime} \cup L^{\sharp}$ in the notation of section 6.1. The goal is to show that this link is h-trivial. To achieve this, the stabilization will require a slight modification, based on the observation in remark 3.3. This argument is independent of section 6.1; it can be carried out in the setting of [7].

Consider the links in figure 6.4. They are obtained from the elementary Engel links in figure 3.1 by adding an additional component labeled $g_{i}$ in the figure. These links are geometric realizations of 5 -fold almost basic commutators $\left[h_{1}, \ldots, h_{5}\right]$ in the statement of corollary 3.2, where the first element $h_{1}$ is a generator $g_{i}$, rather than a product of two generators. Geometrically this is manifested in the fact that the band sums used to construct the links do not involve the component labeled $g_{i}$. As pointed out in remark 3.3, any element $g \in \pi^{k}, k \geq 5$, may be represented in the Milnor group $M \pi$ as a product of conjugates of commutators of this type.

(a)

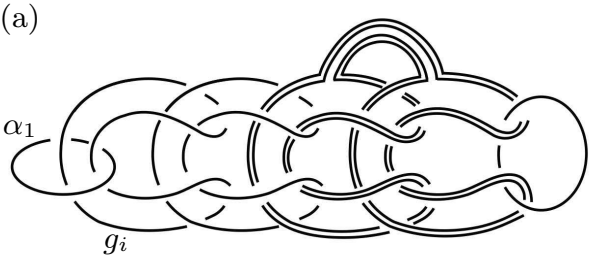

(b)

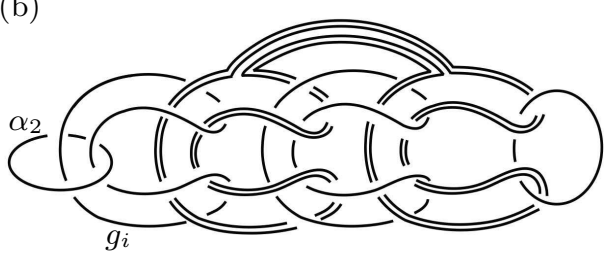

FiguRE 6.4. Links in figure 3.1 with an extra component. (a): $\alpha_{1}=$ $\left[g_{i}, \gamma_{1}\right], \quad(\mathrm{b}): \alpha_{2}=\left[g_{i}, \gamma_{2}\right]$.

To illustrate the use of the links in figure 6.4, first consider the Borromean rings with a parallel copy $l_{0}^{\prime}$ of one of its components $l_{0}$, figure 6.5. Denoting by $m_{1}, m_{2}$ the meridians suitably connected to a basepoint and similarly regarding $l_{0}$ as a based 
loop, note that the expression $l_{0}=\left[m_{1}, m_{2}\right]$ holds regardless of whether $l_{0}$ is considered as an element of the Milnor group $M \pi_{1}\left(S^{3} \backslash\left(l_{1} \cup l_{2}\right)\right)$ or as an element of $M \pi_{1}\left(S^{3} \backslash\left(l_{0}^{\prime} \cup l_{1} \cup l_{2}\right)\right)$. This expression can be read off from the torus bounded by $l_{0}$ in the complement of $l_{1}, l_{2}$, figure 6.5.

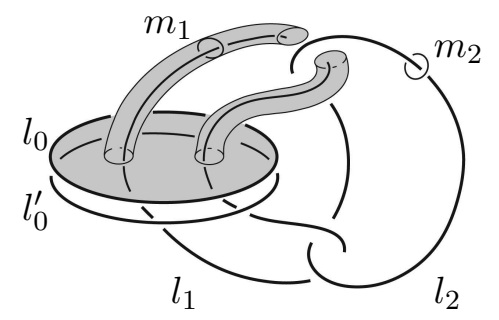

FigURE 6.5.

Similarly, the expressions read off by the components $\alpha_{i}$ of the links in figure 6.4 are unchanged when a parallel copy of $\alpha_{i}$ is present. This calculation may be given in terms of the Milnor group, or it can be read off from a grope bounded by $l_{0}$ in the complement of the link. The links in question may be represented as a "composition", in the sense of [8, Theorem 2.3], where the components labeled $\alpha$ and $g_{i}$ of a link in figure 6.4 are identified with the components $l_{0}, l_{1}$ of the Borromean rings in figure 6.5, and the rest of the link (denote it by $Q$ ) is inserted in a solid torus neighborhood $T$ of $l_{2}$. (This may also be understood as an instance of the more general satellite construction.) The following argument applies to any link $Q$ in $T$. As in figure 6.5,

$$
l_{0}=\left[m_{1}, m_{2}\right] .
$$

Consider the meridian $m_{2}$ as an element in the Milnor group of the complement of $Q$ in the solid torus $T, M \pi_{1}(T \backslash Q)$. The generators of this Milnor group are meridians to $Q$ and a longitude of the solid torus. Considered as part of figure 6.5, this longitude represents the commutator $\left[m_{1}, m_{0} m_{0}^{\prime}\right]$. Substituting this into (6.2), observe that the only way that the meridian $m_{0}^{\prime}$ appears in the expression for $l_{0}$ is as part of the commutator

$$
\left[m_{1}, \ldots \cdot\left[m_{1}, m_{0} m_{0}^{\prime}\right] \cdot \ldots\right] .
$$

Applying the commutator identities (5.3) and the Milnor relation (3.1), it follows that omitting $\left[m_{1}, m_{0} m_{0}^{\prime}\right]$ from this expression does not change the element in the Milnor group, establishing the desired claim.

Now consider a GBR of the form $l_{0} \cup \bar{L}$, where $l_{0}$ is a 5 -fold commutator in the complement of $\bar{L}$. (For example in figure 6.1 Bing double any one of the components $l_{2}, \ldots, l_{4}$.) Using remark 3.3, the construction in section 6.1 goes through without any changes, except that the elements $\alpha_{i}, \beta_{i}, i=1,2$ in the link $l \cup L$ in figure 6.3 (b) are 5-fold (rather than 4-fold) commutators. The elementary Engel links used to build $K_{0}$ are now of the form in figure 6.4 (with a parallel copy of the band-summed curves removed), rather than figure 6.2. 
Given such GBR $l_{0} \cup L$ and stabilization $K_{0}, l_{0}$ then may be assumed to be in the subgroup (isomorphic to $M \pi_{1}\left(S^{3} \backslash\left(L \cup K_{0}\right)\right)$ ) of $M \pi_{1}\left(S^{3} \backslash\left(l_{0}^{\prime} \cup L \cup K_{0}\right)\right.$ ), generated by meridians to $L \cup K_{0}$. As in the proof in section 6.1, only the homological information about bands connecting $L$ and $K_{0}$ and forming $L^{\sharp}$ is relevant. Choose arcs connecting $L$ with $K_{0}$ so that the corresponding conjugating elements are in the subgroup $M \pi_{1}\left(S^{3} \backslash\left(L \cup K_{0}\right)\right)$ of $M \pi_{1}\left(S^{3} \backslash\left(l_{0}^{\prime} \cup L \cup K_{0}\right)\right)$. Now all calculations in the preceding sections, establishing that $l_{0}=1 \in M \pi_{1}\left(S^{3} \backslash L^{\sharp}\right)$, do not involve the meridian to $l_{0}^{\prime}$, so

$$
l_{0}=1 \in M \pi_{1}\left(S^{3} \backslash\left(l_{0}^{\prime} \cup L^{\sharp}\right)\right) .
$$

The link $l_{0}^{\prime} \cup L^{\sharp}$ is h-trivial, therefore its Milnor group is the free Milnor group, and (6.3) is equivalent to h-triviality of the link $l_{0} \cup l_{0}^{\prime} \cup L^{\sharp}$. This concludes the proof of theorem 1.

Acknowledgments. VK was partially supported by NSF grant DMS-1309178. He also would like to thank the IHES for hospitality and support (NSF grant 1002477).

\section{REFERENCES}

[1] W. Burnside, On groups in which every two conjugate operations are permutable, Proc. Lond. Math. Soc. 35 (1902), 28-37.

[2] T.D. Cochran, Derivatives of links: Milnor's concordance invariants and Massey's products, Mem. Amer. Math. Soc. 84 (1990), no. 427.

[3] M. Freedman, The topology of four-dimensional manifolds, J. Differential Geom. 17(1982), 357453.

[4] M. Freedman, The disk theorem for four-dimensional manifolds, Proc. ICM Warsaw (1983), 647-663.

[5] M. Freedman, A geometric reformulation of four dimensional surgery, Topology Appl., 24 (1986), 133-141.

[6] M. Freedman, Are the Borromean rings (A,B)-slice?, Topology Appl., 24 (1986), 143-145.

[7] M. Freedman and V. Krushkal, Engel relations in 4-manifold topology, Forum Math. Sigma 4 (2016), e22, 57 pp. arXiv:1412.5024

[8] M. Freedman and X.S. Lin, On the $(A, B)$-slice problem, Topology Vol. 28 (1989), 91-110.

[9] M. Freedman and F. Quinn, The topology of 4-manifolds, Princeton Math. Series 39, Princeton, NJ, 1990.

[10] M. Freedman and P. Teichner, 4-Manifold Topology I: Subexponential groups, Invent. Math. 122 (1995), 509-529.

[11] M. Freedman and P. Teichner, 4-Manifold Topology II: Dwyer's filtration and surgery kernels, Invent. Math. 122 (1995), 531-557.

[12] C. Hopkins, Finite groups in which conjuate operations are commutative, Am. J. Math. 51, (1929), 35-41.

[13] V. Krushkal, Additivity properties of Milnor's $\bar{\mu}$-invariants, J. Knot Theory Ramifications 7 (1998), 625-637.

[14] V. Krushkal, A counterexample to the strong version of Freedman's conjecture, Ann. of Math. 168 (2008), 675-693.

[15] V. Krushkal and F. Quinn, Subexponential groups in 4-manifold topology, Geom. Topol. 4 (2000), 407-430. 
[16] F.W. Levi, Groups in which the commutator operation satisfies certain algebraic conditions, J. Indian Math. Soc. 6 (1942), 87-97.

[17] W. Magnus, A. Karrass and D. Solitar, Combinatorial group theory: Presentations of groups in terms of generators and relations, Interscience Publishers, New York-London-Sydney 1966.

[18] J. Milnor, Link Groups, Ann. Math 59 (1954), 177-195.

[19] J. Milnor, Isotopy of links, Algebraic geometry and topology, Princeton Univ. Press, 1957, 280-306.

Microsoft Station Q, University of California, Santa Barbara, CA 93106-6105, and Department of Mathematics, University of California, Santa Barbara, CA 93106

E-mail address: michaelf@microsoft.com

Department of Mathematics, University of Virginia, Charlottesville, VA 22904

E-mail address: krushkal@virginia.edu 